\title{
Technique description: Incisionless ultrasound-assisted biceps tenotomy in dogs
}

\author{
David Lane ${ }^{1^{*}}$ and Teresa Schiller ${ }^{2}$
}

\author{
${ }^{1}$ Points East West Veterinary Services, Box 2696, Garibaldi Highlands, British Columbia, V0N 1T0, Canada \\ ${ }^{2}$ Faculty of Veterinary Medicine, University of Calgary, 11877 85th St NW, Calgary, Alberta, T3R 1J3, Canada
}

\begin{abstract}
Background: Bicipital tendinitis and/or tendinopathy is a common cause of forelimb lameness in dogs, particularly in larger and more active patients. Although conservative treatment aimed at resolving discomfort and preserving the tendon remains the primary therapeutic goal, in certain cases it is necessary to surgically transect the tendon to eliminate pain and lameness. Transection of the tendon can be performed by open arthrotomy, arthroscopically, or percutaneously using a scalpel blade. This paper examines the utility of a modified percutaneous approach using a hypodermic needle in place of a scalpel blade, under ultrasound-guided assistance.

Aim: To develop and describe a surgical technique for performing a percutaneous biceps tenotomy using a hypodermic needle under ultrasound guidance

Method: The technique was piloted using the shoulders of 12 cadaver dogs initially and once developed, then applied to another 12 cadavers. The final procedure was performed on a total of 22 shoulders. Assessments were recorded on time to complete the procedure, completeness of bicipital tendon transection, and presence of any iatrogenic damage to associated joint structures.

Results: Procedure time averaged fewer than 2 minutes. Complete transection was achieved in 20 out of 22 of the shoulders, with evidence of incomplete transection discernable by ultrasound imaging in the remaining two shoulders. One cadaver shoulder experienced iatrogenic damage secondary to incorrect hypodermic needle angulation.

Conclusion: Percutaneous biceps tenotomy using a hypodermic needle is an efficient and straightforward procedure. The lack of a surgical incision makes it the least invasive technique devised so far. Ultrasound imaging allows the practitioner to assess the completeness of the transection increasing precision.

Keywords: Biceps, Canine, Percutaneous, Tendinopathy, Tenodesis.
\end{abstract}

\section{Introduction}

The biceps tendon originates from the supraglenoid tubercle of the scapula, spans the glenohumeral joint, entering the intertubercular groove of the proximal humerus before transitioning into a pennate muscle, inserting the intra-articular at the distal aspect of the medial collateral ligament and elbow joint capsule attachment (Wall and Taylor, 2002; Wernham et al., 2008). The biceps muscle's primary action is elbow flexion, but it also aids in both shoulder extension and stabilization (Sidaway et al., 2004; Wernham et al., 2008).

Injury to the biceps tendon of origin can be the result of acute overloading or secondary to repetitive stress, and is typically seen in active, middle-aged, or older large breed dogs. The severity of lameness varies, ranging from subtle decreases in performance to profound weight bearing lameness (Millis and Levine, 2013; Leeman et al., 2016; Zink and Van Dyke, 2018). Pain on palpation along the tendon length, especially with concurrent shoulder flexion and elbow extension, is a common physical examination finding (Millis and Levine, 2013; Leeman et al., 2016; Zink and Van Dyke, 2018). Diagnosis is made by combining physical examination findings with imaging results, or with arthroscopic assessment (Millis and Levine, 2013; Zink and Van Dyke, 2018). Radiography is poorly sensitive for shoulder injuries and is inadequate for identifying lesions of the biceps tendon itself, although contrast radiography may yield more specific results (Wernham et al., 2008). Ultrasound is being used more frequently as a diagnostic tool (Cook, 2016). Relative to magnetic resonance imaging or arthroscopy, ultrasound assessment is more accessible, and less expensive, especially when serial imaging is required (Cook, 2016).

Bicipital tenosynovitis describes a condition in which there is evidence of inflammation of the sheath surrounding the biceps tendon (Davidson et al., 2000). This condition can be secondary to supraspinatus tendinopathy, wherein the supraspinatus tendon enlarges and encroaches into the bicipital groove, and impinges upon the bicipital sheath (Lafuente et al., 2009). This encroachment can result in a focal narrowing, displacing the biceps tendon, creating friction, and resulting in inflammation (Fransson et al., 2005).

When addressing bicipital tendon tears or tenosynovitis, conservative therapy still remains the treatment of 
choice, as it preserves the tendon and retains function (Millis and Levine, 2013; Leeman et al., 2016; Zink and Van Dyke, 2018). Such options include analgesics, cryotherapy, exercise restriction, therapeutic ultrasound, extracorporeal shockwave therapy, laser, massage, intra-articular corticosteroids, intra-articular platelet-rich plasma, and stem cell therapy (Millis and Levine, 2013; Leeman et al., 2016; Zink and Van Dyke, 2018). To date, there is limited data on the effectiveness of these treatments. Leeman et al. (2016) demonstrated that $85 \%$ of the patients receiving extracorporeal shockwave therapy for either biceps, supraspinatus, or combined biceps and supraspinatus tendon pathology, had good or excellent outcomes based on subjective owner assessment. Although regenerative medicine techniques incorporating cultured stem cells, bone marrow aspirate concentrate, or adipose-derived stromal vascular fraction have not been investigated as a treatment for biceps tendon pathology, they have shown positive outcomes when used to address either canine supraspinatus tendon or cruciate ligament injury (Canapp et al., 2016a, 2016b). This paper's primary author has experienced positive outcomes using combined platelet-rich plasma and bone marrow aspirate concentrate when addressing biceps tendon pathology (Figs. 1 and 2).

Patients that fail to respond to conservative treatment are candidates for surgical therapy, which consists of either surgical tenodesis or tendon release (tenotomy) via complete transection of the remaining tendon (Wall and Taylor, 2002; Cook et al., 2005; Esterline et al., 2005; Wernham et al., 2008; Leeman et al., 2016). Tenodesis is recommended in athletic dogs by some, despite the increased complication rate of this procedure relative to a release procedure (Wernham et al., 2008). Both these surgical options result in the biceps tendon no longer spanning the glenohumeral joint, and instead retracting to the proximal humerus region where it is thought to adhere to adjacent structures (Wall and Taylor, 2002;

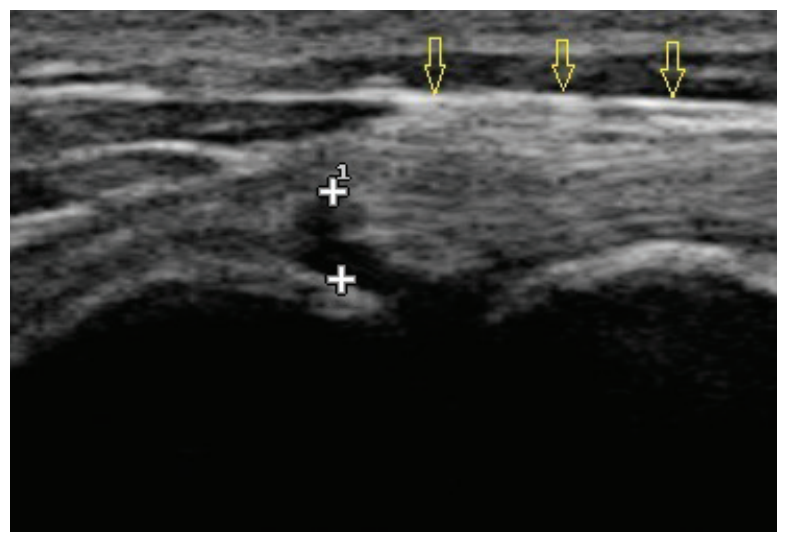

Fig. 1. Longitudinal ultrasound image of an acutely torn biceps tendon (yellow arrows). The tear itself can be seen as an absence of fibers between the two "+" symbols.
Wernham et al., 2008). Consequently, the patient no longer benefits from the tendon's shoulder extension or stabilization functions. Multiple researchers (Bardet, 1998; Esterline et al., 2005; Wernham et al., 2008) report a belief that the biceps tendon does not significantly contribute to glenohumeral stability based on Vasseur et al.'s (1982) qualitative research. However, subsequent quantitative research determined that the biceps tendon does contribute to passive glenohumeral stabilization, and that bicipital tendon transection generates significant glenohumeral laxity (Sidaway et al., 2004). Although the degree to which the biceps contribute to active glenohumeral stability is unknown, Cullen et al. (2016) demonstrated that jumping causes a 5.5 times increase in biceps muscle activation relative to walking, ascending ramps causes a 5.1 times increase, and descending ramps a 4.8 times increase.

The long-term outcomes for bicipital tendon transection yields favorable results based on subjective owner assessment, and only limited or no progression of radiographic degenerative changes (Bergenhuyzen et al., 2010). The long-term outcome for tenodesis has not been examined (Wall and Taylor, 2002; Cook et al., 2005; Wernham et al., 2008; Bergenhuyzen et al., 2010), but the prognosis for short-term reduction of pain and lameness has been shown to be good to excellent (Wall and Taylor, 2002; Cook et al., 2005; Esterline et al., 2005; Wernham et al., 2008; Leeman et al., 2016).

These procedures can be performed by either an open surgical approach, via arthroscopy, or by a percutaneous approach using a scalpel blade (Millis and Levine, 2013; Zink and Van Dyke, 2018). An open arthrotomy is considered the most invasive approach as it requires transection of the superficial pectoral muscle via an incision that runs from the acromial process to the mid humerus (Johnson, 2013). Arthroscopy using two or three ports is considerably less invasive but requires

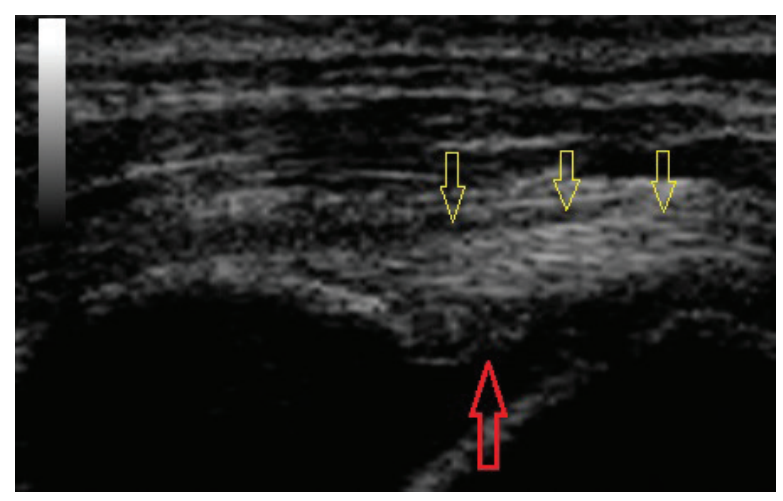

Fig. 2. The same biceps tendon as Figure 1 (yellow arrows) 8 weeks following intralesional injection with bone marrow aspirate concentrate and platelet-rich plasma. Significant fiber infilling of the previous lesion can be seen. 
expensive instrumentation and surgical experience that likely requires referral to a specialist facility.

Esterline et al. (2005) used cadavers to develop a technique using a number 10 scalpel blade to make a $2-\mathrm{cm}$ incision through the skin, and a number 12 blade to make a $1-\mathrm{cm}$ incision through the muscle, before transecting the tendon itself (Esterline et al., 2005). The use of ultrasound imaging, rather than a blind approach, resulted in a longer procedure time (53 \pm 8 seconds vs. $209 \pm 74$ seconds) and a slightly longer skin incision length $(2.04 \pm 0.1 \mathrm{~cm} v s .1 .13 \pm 0.5 \mathrm{~cm})$, but also improved success in completely transecting the tendon. Using the blind technique, 5 out of 11 tendons were incompletely transected, but only 1 of 11 of the ultrasound-assisted tendons were incompletely transected. This technique was subsequently adopted by Peppler et al. (2009) and applied to five clinical cases, with short-term success rates mirroring that of an arthroscopic tendon release.

Despite the disadvantages to performing a bicipital tenotomy or tenodesis, it remains a viable option for situations in which the tendon fails to respond to other treatments, or is otherwise deemed unsalvageable. However, the invasiveness of an open approach, or accessibility to arthroscopy may prove to be a barrier to some clients. In these situations, a percutaneous approach may be the best therapeutic option.

We hypothesized that the percutaneous tenotomy technique proposed by Esterline et al. (2005) could be improved upon; that under ultrasound guidance, a hypodermic needle could be used to completely transect the biceps tendon without the need of tissue incisions or a scalpel blade. Such a procedure would have the advantages of requiring no specialized surgical equipment, could be the least invasive approach, and could where indicated, be performed at the time of ultrasound diagnosis for cases for which the tendon is deemed unsalvageable.

\section{Materials and Methods}

Twenty-four canine cadavers were obtained through the Faculty of Veterinary Medicine, University of Calgary. An initial batch of 12 dogs was used to develop the final technique. All ultrasound imaging was performed using a Sonoscape S9 Portable Digital Color Doppler Ultrasound System, China, with a $12 \mathrm{MHz}$ linear probe. Attempts were made to transect the biceps tendon from its origin on the supraglenoid tubercle, and at the point where it spanned the glenohumeral joint space. The former location was deemed to be technically demanding under ultrasound guidance, and the biceps tendon was so lax at the latter location that it frequently rolled off the bevel of the needle, impeding transection. Attempts to transect the biceps tendon as it traversed the intertubercular groove were more successful, so this became the location for the final technique.

To test the final technique, additional 12 dogs were acquired under the same circumstances as the original batch. The procedure was performed 11 times by each researcher. For 10 of the dogs, one researcher performed the procedure on the left bicipital tendon and the other researcher performed the procedure on the right bicipital tendon. The right shoulder of the first dog was used as a "practice run", and was not included in the research data. The left proximal humerus of the twelfth dog was grossly enlarged, consistent in appearance and texture with a proliferative osteosarcoma, so it too was excluded. Following completion of the procedure for each shoulder, an immediate dissection was performed to assess completeness of the biceps tendon transection, and to look for any iatrogenic damage to the associated soft tissue or humerus.

Each dogs' weight and sex was recorded, as was the time to complete each transection. The completeness of the transection and evidence of any iatrogenic damage were also noted. Basic statistical analysis was performed. A description of the final procedure is as follows:

The dog was placed in lateral recumbency with the affected limb uppermost. Hair was clipped to expose the humeral tubercle and intertubercular groove. Using a $12 \mathrm{mHz}$ linear probe, the biceps tendon was visualized longitudinally from the musculotendinous junction within the groove, proximally to the tendinous origin on the supraglenoid tubercle (Fig. 3). Any abnormalities and the approximate tendon size were noted. The biceps tendon was then positioned under tension by placing the shoulder in flexion with concurrent elbow extension, so that the forelimb lay roughly parallel to the topline. With the limb in this position, the appearance of the biceps tendon as it spanned the glenohumeral joint was noted, and images were saved to the ultrasound's hard drive.

The ultrasound probe was removed and the biceps tendon palpated along the bicipital groove distally to its transition into biceps muscle. An assistant was used to restrain the limb in the biceps tension position, with concurrent humeral external rotation. A hypodermic

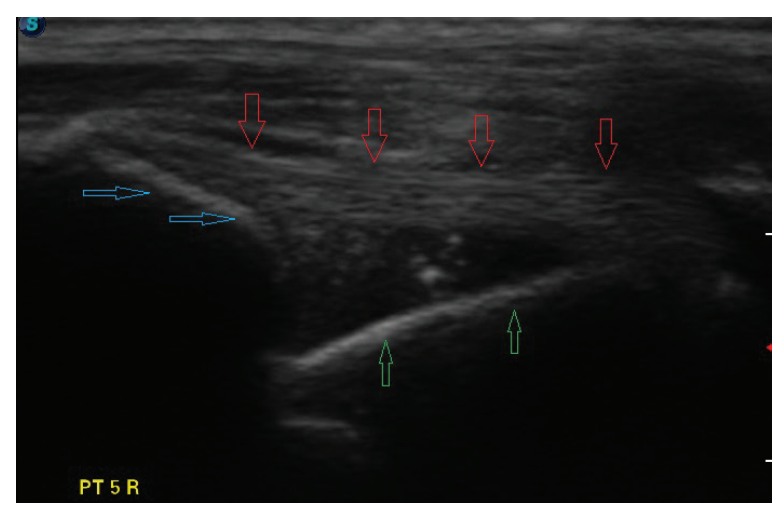

Fig. 3. Longitudinal ultrasound image of an intact biceps tendon (red arrows), supraglenoid tubercle (blue arrows), and proximal humerus (green arrows). 
needle, $18 \mathrm{~g}$ for toy dogs, and $16 \mathrm{~g}$ for larger dogs, was inserted perpendicular to the humeral surface, just distal from the most cranial aspect of the greater tubercle, onto the medial margin of the bone. With the beveled edge pointed in a mediolateral direction, the needle was pulled laterally from its starting position medial to the biceps tendon. For thicker tendons, the needle needed to be retracted from the bone surface for initial cuts, and was then advanced deeper as the tendon transection progressed. Eventually, the tip of the bevel of the needle needed to contact the underlying bone across the entire bicipital groove to ensure a complete transection. Medial to lateral cuts were repeated until the tendon was transected.

As the transection progressed, the assistant was often able to detect increasing laxity, and needed to progressively flex the shoulder further to maintain consistent tension on the remaining biceps tendon. An abrupt increase in shoulder flexion frequently accompanied complete transection of the tendon.

Once it was subjectively felt the transection was complete based on palpation, a repeat ultrasound image of the biceps tendon was obtained to determine the completeness of the transection. The criterion used to determine complete transection of the tendon based on palpation included feeling an increased range of motion during shoulder flexion, by palpating an absence of biceps tendon in the bicipital groove, and if possible by palpating the distal edge of the transected tendon. The ability to clearly palpate the tendon or its transected edge varied with the size and musculature of the dog.

Ultrasound verification of complete tendon transection was demonstrated if the tendon remained lax proximally as it spanned the glenohumeral joint, even though the limb position had not changed (Fig. 4). The tendon's appearance, following transection, could be compared to the images taken earlier, as needed. Longitudinal and transverse imaging was also performed over the transection site to demonstrate a complete absence of tendon fibers (Fig. 5). If incomplete transection

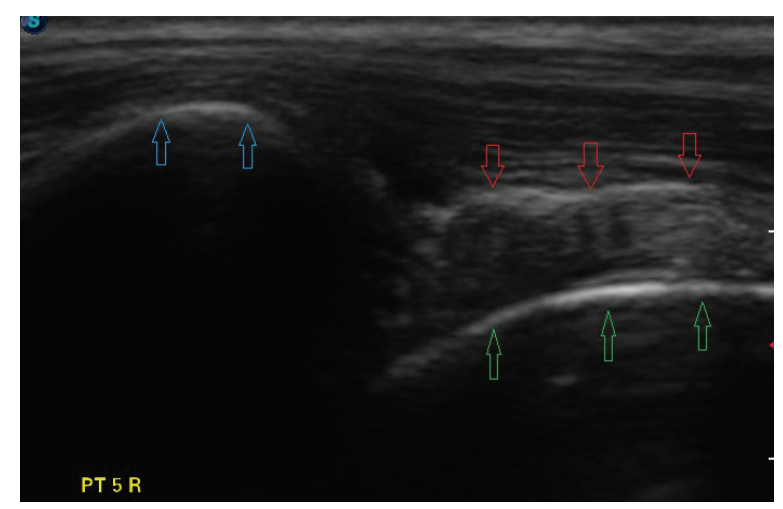

Fig. 4. Longitudinal ultrasound image of a biceps tendon (red arrows) post-transection, supraglenoid tubercle (blue arrows), and proximal humerus (green arrows). Note the laxity now present. was observed, the ultrasound probe was removed and the procedure repeated until complete transection occurred. On two occasions there was discordance between the second researcher's assessment of the complete transection based on palpation, compared to ultrasound findings. On both those occasions, it was elected to proceed with dissection rather than resume the transection.

The study was reviewed and approved by the Veterinary Sciences Animal Care Committee (Protocol AC190177 VetMed 320) and involved a secondary use of tissues from 24 canine cadavers, ethically procured and used for a reviewed and approved educational purpose.

\section{Results}

Twenty-two biceps tendons were transected on 12 dogs. All dogs were female and weighed an average of $28.7 \mathrm{~kg}$ (range: $14.7-43.6 \mathrm{~kg}$ ). Breed could not be accurately determined, so it is unknown if any purebred dogs were represented, although two dogs looked like golden retrievers, and one appeared to be a whippet. The remaining dogs appeared to be Retriever, German shepherd, or Staffordshire terrier crosses.

Average time to complete tendon transection, defined as the span of time from when the researcher unsheathed the hypodermic needle until the moment it was felt that the tendon was completely transected, averaged $105 \pm 97$ seconds (range: $27-456$ seconds). The first researcher averaged 83 seconds (range: 27-186 seconds) and the second researcher averaged 126 seconds (range: 28-456 seconds). The time to transection did not include the time spent repeat imaging the tendon using the ultrasound probe. If, after repeat imaging, it was determined that the tendon was not completely transected, then the timer was started again, ceasing once more when the transection was deemed complete. A complete list of patient signalment and transection times is presented in Table 1.

Complete biceps tendon transection occurred in 20 of 22 shoulders. Of the two shoulders in which transection was incomplete, evidence of incomplete transection was

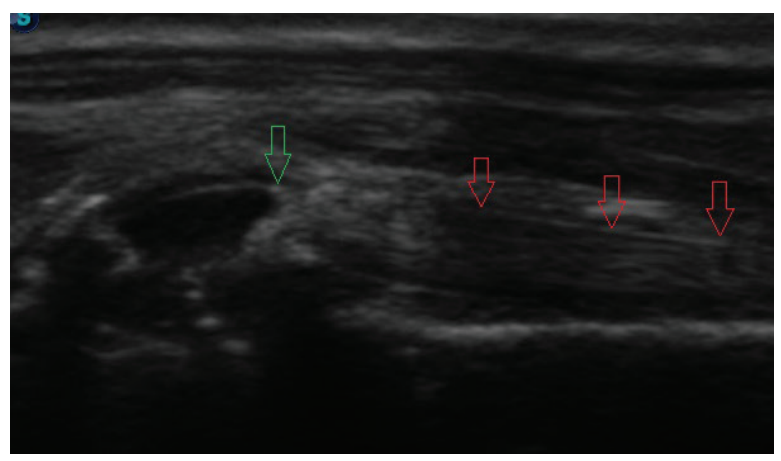

Fig. 5. Longitudinal ultrasound image of a transected biceps tendon. The intact tendon is indicated with red arrows and the point of transection with a green arrow. 
Table 1. Patient weight, procedure time, and outcome.

\begin{tabular}{|c|c|c|c|c|c|}
\hline ID number & Side & Researcher & BW (kg) & Procedure time (seconds) & Result \\
\hline \multirow{2}{*}{1} & Right & & \multirow{2}{*}{27.6} & Not recorded & Not included as part of study \\
\hline & Left & 1 & & 186 & Complete \\
\hline \multirow{2}{*}{2} & Right & 1 & \multirow{2}{*}{23.5} & 43 & Complete \\
\hline & Left & 2 & & 45 & Complete \\
\hline \multirow{2}{*}{3} & Right & 1 & \multirow{2}{*}{31.7} & 74 & Complete \\
\hline & Left & 2 & & 222 & Incomplete - small remnant \\
\hline \multirow{2}{*}{4} & Right & 2 & \multirow{2}{*}{43.6} & 456 & incomplete $-20 \%$ functional remnant \\
\hline & Left & 1 & & 180 & Complete \\
\hline \multirow{2}{*}{5} & Right & 2 & \multirow{2}{*}{25.9} & 150 & Complete \\
\hline & Left & 1 & & 30 & Complete \\
\hline \multirow{2}{*}{6} & Right & 1 & \multirow{2}{*}{21.5} & 30 & Complete \\
\hline & Left & 2 & & 120 & Complete \\
\hline \multirow{2}{*}{7} & Left & 1 & \multirow{2}{*}{24} & 27 & Complete \\
\hline & Right & 2 & & 55 & Complete \\
\hline \multirow{2}{*}{8} & Right & 1 & \multirow{2}{*}{33.4} & 45 & Complete \\
\hline & Left & 2 & & 90 & Complete \\
\hline \multirow{2}{*}{9} & Left & 1 & \multirow{2}{*}{14.7} & 85 & Complete \\
\hline & Right & 2 & & 68 & Complete \\
\hline \multirow{2}{*}{10} & Right & 1 & \multirow{2}{*}{36.5} & 145 & Complete - iatrogenic damage \\
\hline & Left & 2 & & 56 & Complete \\
\hline \multirow{2}{*}{11} & Right & 2 & \multirow{2}{*}{22.3} & 28 & Complete \\
\hline & Left & 1 & & 64 & Complete \\
\hline \multirow{2}{*}{12} & Right & 2 & \multirow{2}{*}{39.5} & 100 & Complete \\
\hline & Left & & & Not recorded & Rejected from study due to local pathology \\
\hline
\end{tabular}

evident on repeat ultrasound. The left biceps of the third dog had a small tag of lax friable tendon remaining. This tissue had a striated appearance on ultrasound consistent with tendon, rather than paratendon sheath. Tension applied transversely by hemostats easily ruptured this remaining fragment, and it was subjectively thought that it would not have affected the outcome of an actual patient (Fig. 6). The right tendon of the fourth dog was only about $80 \%$ transected, with deeper portions of the tendon remaining. The deeper portion of remaining tendon was evident on ultrasound examination prior to the dissection. Subjectively, it was thought that this amount of residual tendon would likely cause ongoing pain in a real patient.

One shoulder experienced iatrogenic damage during the bicipital transection; partway through the procedure, the first researcher realized the needle was angled cranially rather than perpendicular to the long axis of the humerus. The needle angle was corrected and the procedure was completed successfully, but upon dissection it was determined that the cartilage surface of the humeral head had multiple full thickness

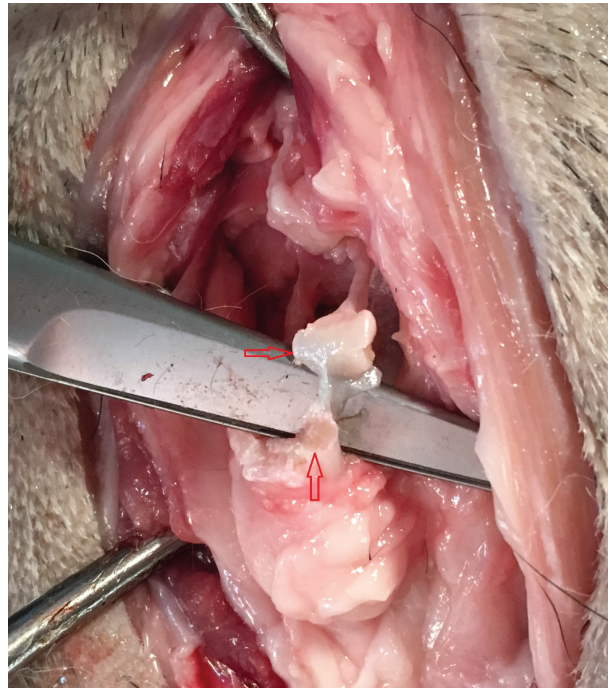

Fig. 6. Persistent remnant of tissue following incomplete transection. This fragment was friable and thought not to likely diminish the procedure outcome in an actual clinical case. 
lacerations (Fig. 7). Additionally, there was a $2-3 \mathrm{~mm}$ laceration of the medial aspect of the supraspinatus tendon (Fig. 8). Superficial scoring of the cartilage of the bicipital groove by the tip of the needle was evident in all shoulders at the level of the transection.

\section{Discussion}

The beveled edge of a 16 gauge hypodermic needle, aided by ultrasound imaging, appears to be an effective tool for performing a bicipital tenotomy at the level of the intertubercular groove. The procedure can be performed rapidly, averaging fewer than 2 minutes to complete, and without the need for an incision.

Complications occurred in 3 of 22 shoulders (13.6\%). Two shoulders experienced incomplete transection of the biceps tendon, although the amount of tendon remaining in one of those shoulders was deemed unlikely to be clinically significant. On both occasions, the portion of remaining tendon was evident on ultrasound. Determining the completion of the procedure based on ultrasound findings, rather than by palpation, should reduce the chance of this complication.

One of the 22 shoulders experienced iatrogenic damage to the humeral articular cartilage as a result of the needle being angled cranially, rather than perpendicular to the surface of the humerus. Remaining cognizant of the needle orientation and anatomic location, via meticulous land marking, should prevent this complication.

The location of the bicipital transection with this procedure is slightly distal to the location of bicipital transection performed arthroscopically (Wall and Taylor, 2002; Wernham et al., 2008). Variation in transection location for arthroscopic transection has been described, with no known optimal location reported (Wernham et al., 2008). Our approach was similar to that employed by Peppler et al. (2009) who reported outcomes that mirrored those produced by arthroscopic tenotomy.

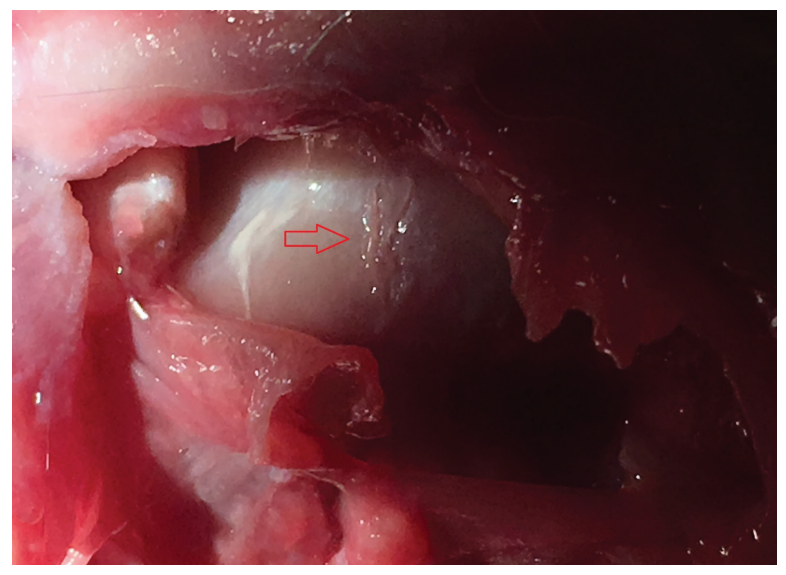

Fig. 7. Iatrogenic laceration of the cartilage of the humeral head (red arrow).
Scoring of the cartilage of the bicipital groove was evident in all shoulders at the level of the transection. Since the end result of the procedure is that the biceps tendon will no longer glide within the groove at the level of this scoring, the authors suspect that this lesion will not negatively affect the outcome of the procedure. Confirmation of this suspicion can only be achieved by monitoring the results after performing the procedure on naturally occurring clinical cases, and comparing outcomes relative to arthroscopic release.

This paper is limited in that it is a cadaveric study. Consequently, no conclusions can be made about invivo clinical outcomes of this procedure. Additionally, all the 22 tendons appeared normal based on ultrasound assessment. It is unknown if transection would be more difficult in biceps tendons with naturally occurring pathology, relative to healthy tendons. This limitation can only be overcome through clinical application on naturally occurring cases of bicipital tendinopathy.

The advantages of this procedure over open arthrotomy, arthroscopic transection, or percutaneous transection using as scalpel blade, are that the need for an incision is eliminated, potentially decreasing post-operative morbidity and incision-related complications; that no expensive arthroscopic instrumentation or training is required; and that the shorter procedure time and lack of incision means a reduction in anesthesia time and potential complications. The primary author has successfully performed this procedure twice on clinical patients using a combination of intravenous hydromorphone and dexmedetomidine, combined with intra-articular mepivacaine. These advantages will likely reduce the procedure cost for the client, potentially making the procedure more accessible.

An additional advantage of this technique is that ultrasound-guided percutaneous transection can potentially be performed more rapidly than either an open or arthroscopic approach. Esterline et al. (2005)

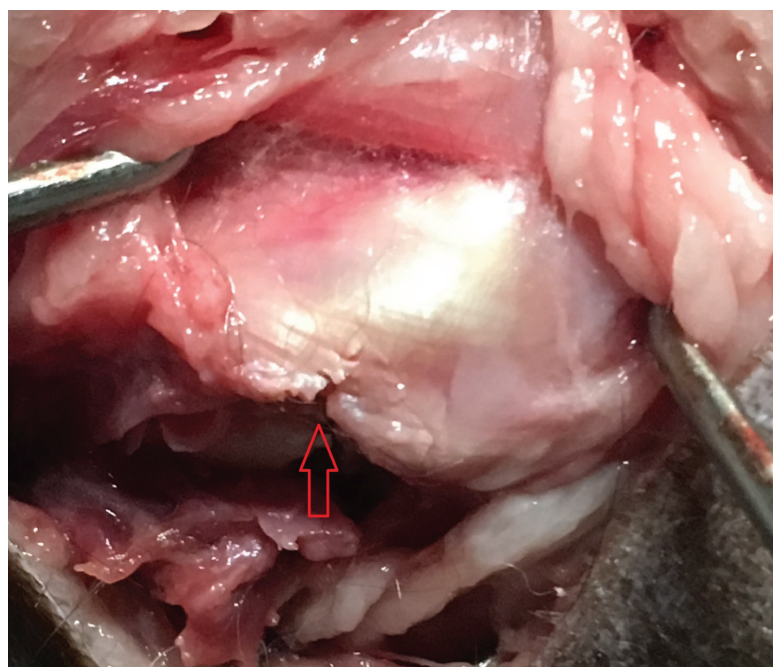

Fig. 8. Iatrogenic laceration of the supraspinatus tendon (red arrow). 
reported an average procedure time of $53 \pm 8$ seconds without ultrasound assistance, and $209 \pm 74$ seconds with ultrasound assistance. However, when Peppler et al. (2009) performed this procedure in actual clinical cases, they reported an average procedure time of 16 minutes (960 seconds). Because the procedure time reported in our paper did not include the time spent performing a repeat ultrasound assessment, no direct comparison can be made with the time taken by Peppler et al. (2009) or by Esterline et al. (2005) with ultrasound guidance. Our methodology for measuring the time taken most closely matches Esterline et al.'s (2005) technique without ultrasound guidance, which also took fewer than 2 minutes to complete.

Because surgical transection of the biceps tendon will result in subsequent destabilization of the glenohumeral joint (Sidaway et al., 2004), it should be considered a salvage procedure, only to be attempted when conservative therapeutic measures fail, or the tendon is otherwise deemed unsalvageable. In cases where biceps tenosynovitis is secondary to impingement from an enlarged supraspinatus tendon, treatment should be directed toward the supraspinatus tendon itself. The biceps aids the supraspinatus in extending the glenohumeral joint; if supraspinatus pathology exists, it remains unproven whether or not removing the stabilizing and shoulder extension functions of the biceps via a tenotomy, will increase the physiologic demands on the supraspinatus tendon and subsequently contribute to its ongoing pathology.

Although many researchers list exercise restriction, non-steroidal anti-inflammatories, and/or intra-articular corticosteroid injections as a their treatment of choice for biceps tendon pathology or tenosynovitis, other treatments such as extracorporeal shockwave or regenerative medicine may prove to have a higher success rate, and therefore should be considered before electing tendon transection (Wernham et al., 2008; Millis and Levine, 2013; Leeman et al., 2016; Zink and Van Dyke, 2018).

In cases where conservative treatment fails, is declined by the client, or if the biceps tendon is otherwise deemed unsalvageable, tenotomy or tenodesis remain the treatments of choice. If a tenotomy is to be performed, ultrasound-assisted percutaneous biceps tendon transection using a hypodermic needle appears to be the most minimally invasive option available.

\section{Conflict of interest}

The authors declare that there are no conflicts of interest.

\section{Authors' contributions}

David Lane: conceptualization, technique development, experiment design, data collection, writing first draft, and edits. Teresa Schiller: technique development, experiment design, data collection, and edits.

\section{References}

Bardet, J. 1998. Diagnosis of shoulder instability in dogs and cats: a retrospective study. J. Am. Anim. Hosp. Assoc. 34, 42-54.
Bergenhuyzen, A.L.R., Vermote, K.A.G., van Bree, H. and Van Ryssen, B. 2010. Long-term followup after arthroscopic tenotomy for partial rupture of the biceps brachii tendon. Vet. Comp. Orthop. Traumatol. 23, 51-5.

Canapp, S.O., Canapp, D.A., Ibrahim, V., Carr, B.J., Cox, C. and Barrett, J.G. 2016a. The use of adiposederived progenitor cells and platelet-rich plasma combination for the treatment of supraspinatus tendinopathy in 55 dogs: a retrospective study. Front. Vet. Sci. 3, 61; doi:10.3389/fvets.2016.00061

Canapp, S.O., Leasure, C.S., Cox, C., Ibrahim, V. and Carr, B.J. 2016b. Partial cranial cruciate ligament tears treated with stem cell and platelet-rich plasma combination therapy in 36 dogs: a retrospective study. Front. Vet. Sci. 3, 112; doi:10.3389/ fvets.2016.00112

Cook, C.R. 2016. Ultrasound imaging of the musculoskeletal system. Vet. Clin. North Am. Small Anim. Pract. 46, 355-71.

Cook, J.L., Kenter, K. and Fox, D.B. 2005. Arthroscopic biceps tenodesis: technique and results in six dogs. J. Am. Anim. Hosp. Assoc. 41, 121-7.

Cullen, K.L., Dickey, J.P., Brown, S.H.M., Nykamp, S.G., Bent, L.R., Thomason, J.J. and Moens, N.M.M. 2016. The magnitude of muscular activation of four canine forelimb muscles in dogs performing two agility-specific tasks. BMC Vet. Res. 13, 68; doi:10.1186/s12917-017-0985-8

Davidson, E., Griffey, S., Vasseur, P. and Shields, S. 2000. Histopathological, radiographic, and arthrographic comparison of the biceps tendon in normal dogs and dogs with biceps tenosynovitis. J. Am. Anim. Hosp. Assoc. 36, 522-30.

Esterline, M.L., Armbrust, L. and Roush, J.K. 2005. A comparison of palpation guided and ultrasound guided percutaneous biceps brachii tenotomy in dogs. Vet. Comp. Orthop. Traumatol. 18, 135-9.

Fransson, B.A., Gavin, P.R. and Lahmers, K.K. 2005. Supraspinatus tendinosis associated with biceps brachii tendon displacement in a dog. J. Am. Vet. Med. Assoc. 227, 1429-33.

Johnson, K.A. 2013. Piermattei's atlas of surgical approaches to the bones and joints of the dog and cat, 5th ed. St. Louis, MO: Elsevier Canada.

Lafuente, M.P., Fransson, B.A., Lincoln, J.D., Martinez, S.A., Gavin, P.R., Lahmers, K.K. and Gay, J.M. 2009. Surgical treatment of mineralized and nonmineralized supraspinatus tendinopathy in twenty-four dogs. Vet. Surg. 38, 380-7.

Leeman, J.J., Shaw, K.K., Mison, M.B., Perry, J.A., Carr, A. and Shultz, R. 2016. Extracorporeal shockwave therapy and therapeutic exercise for supraspinatus and biceps tendinopathies in 29 dogs. Vet. Rec. 179, 385-5.

Millis, D. and Levine, D. 2013. Canine rehabilitation and physical therapy, 2nd ed. Philadelphia, PA: Saunders. 
Peppler, C., Kramer, M. and Gerwing, M. 2009. Ultraschallgestützte perkutane Tenotomie der Bizepssehne als Therapie der Tendo vaginitis bei fünf Hunden: Ein erster Erfahrungsbericht. Tierarztl Prax Ausg K. 37, 167-72.

Sidaway, B.K., McLaughlin, R.M., Elder, S.H., Boyle, C.R. and Silverman, E.B. 2004. Role of the tendons of the biceps brachii and infraspinatus muscles and the medial glenohumeral ligament in the maintenance of passive shoulder joint stability in dogs. Am. J. Vet. Res. 65, 1216-22.

Vasseur, P.B., Moore, D. and Brown, S.A. 1982. Stability of the canine shoulder joint: an in vitro analysis. Am. J. Vet. Res. 43, 352-5.
Wall, C.R. and Taylor, R. 2002. Arthroscopic biceps brachii tenotomy as a treatment for canine bicipital tenosynovitis. J. Am. Anim. Hosp. Assoc. 38, 16975.

Wernham, B.G.J., Jerram, R.M. and Warman, C.G.A. 2008. Bicipital tenosynovitis in dogs. Compend. Contin. Educ. Vet. 30, E8.

Zink, M.C. and Van Dyke, J. 2018. Canine sports medicine and rehabilitation, 1st ed. Hoboken, NJ: John Wiley \& Sons. 